\title{
Multidisciplinary Team to Holistic Integrative Medicine
}

\author{
Zhiping Yang ${ }^{1,2}$ and Daiming Fan ${ }^{2,3 *}$ \\ ${ }^{1}$ Shanghai Mingpin Institute of Holistic Integrative Medicine, Shanghai, China; ${ }^{2}$ China Institute for Development Strategy of Holistic Inte- \\ grative Medicine, Xi'an, China; ${ }^{3}$ State Key Laboratory of Cancer Biology \& National Clinical Research Center for Digestive Diseases, \\ Xijing Hospital of Digestive Diseases, Fourth Military Medical University, Xi'an, China
}

\begin{abstract}
Multidisciplinary teams (MDTs) have been widely used for the diagnosis, treatment and management of cancer patients and other patients with complex diseases and conditions. The decisions of MDTs have not always been the best options as MDTs in general may encourage overtreatment. From MDT to holistic integrative medicine (HIM), a multidisciplinary working model should be established to formulate an individualized and integrated healthcare plan to achieve an optimal effect using a holistic view and integrative thinking.
\end{abstract}

Multidisciplinary teams (MDTs) were introduced in 1995 following evidence of variation in cancer care delivery in the UK, as documented in the Calman-Hine report. ${ }^{1}$ Due to the well-recognized benefits, such as standardization and continuity of care, effective use of resources, improved patient outcomes and the safeguarding of patients from maverick doctors, MDTs have become widely accepted for the diagnosis, treatment and management of cancer patients. ${ }^{1,2}$ The MDT model has since been extended to many other medical fields involving complex diseases and conditions.

However, the decisions emerging from MDTs have not always been the best options. Teo et al. ${ }^{3}$ described the outcomes of 47 patients who underwent surgical resection despite a previous recommendation by a MDT against surgery. From their perspective, it was clear that the decisions made by the local MDTs were inappropriate, and that reversing the multidisciplinary decision changed the prognosis of the patients in 32 out of 47 cases.

The remaining question involves why did this reversal occur? The development of modern medicine is currently facing significant challenges due to the over-specialization and over-division of medical disciplines, as well as the fragmentation of medical knowledge. ${ }^{4}$ Even now, the senior professional within a team is only an expert in a very limited field or specialty. Teamwork has increasingly been used to solve this dilemma. As a knowledge team in health care, a MDT is comprised of a group of diverse health

Keywords: Multidisciplinary team; Holistic integrative medicine; Integrative thinking; Evidence-based medicine; Overtreatment.

Abbreviations: MDT, multidisciplinary team; HIM, holistic integrative medicine; EBM, evidence-based medicine.

Received: November 05, 2020; Revised: November 17, 2020; Accepted: November 18,2020

*Correspondence to: Daiming Fan, China Institute for Development Strategy of Holistic Integrative Medicine; State Key Laboratory of Cancer Biology \& National Clinical Research Center for Digestive Diseases, Xijing Hospital of Digestive Diseases, Fourth Military Medical University, 127\# West Changle Road, Xi'an 710032, China. ORCID: http://orcid.org/0000-0002-2466-5002. Tel: +86-29-84771501, Fax: +86-29-84771466, E-mail: fandaim@fmmu.edu.cn

How to cite this article: Yang Z, Fan D. Multidisciplinary Team to Holistic Integrative Medicine. Exploratory Research and Hypothesis in Medicine 2020;5(4):139140. doi: 10.14218/ERHM.2020.00063. professionals, each contributing to the common goal of providing health care in accordance with his/her competence. ${ }^{5}$ All professionals do their best within the limitations imposed by current scientific approaches and their own knowledge. However, concerted efforts do not necessarily result in positive or optimal healthcare delivery for patients because the extensive procedures do not always have an impact on survival. Sometimes, these procedures can even lead to harmful outcomes and is why iatrogenic factors have become the third leading cause of overall mortality in the USA. ${ }^{6}$

More worrisome is the fact that MDTs may encourage overtreatment. Collective decision making is known to reduce the sense of individual responsibility and to encourage riskier decisions. Under these circumstances, decisions made collectively in MDTs may be biased towards recommending aggressive treatments that have little positive effect but cause patients much misery. ${ }^{7}$ Who makes the final decision at the MDT meeting? It appears that every clinician in a MDT now fears making direct clinical decisions ${ }^{2}$ as they consider the MDT a defensive tool for escaping personal and legal responsibility in achieving a therapy plan.

In order to improve the effectiveness of MDTs, two components are needed: a holistic view and integrative thinking. The human body is an organic being with dynamic changes and powerful selfregulatory and compensation mechanisms. It has been demonstrated that factors such as failure to consider holistic information or patient's views, and a lack of personal knowledge of the patient being discussed, all had an adverse impact on effective clinical decision making in MDTs. ${ }^{8}$ However, holistic thinking looks at the human being as a whole and places patients in a larger context, involving natural, social, psychological, and other factors. In fact, the All of Us Research Program (formerly named Precision Medicine Initiative) aims to determine whole health related factors, including lifestyle, socioeconomic factors, environment, and biologic characteristics to advance precision diagnosis, prevention, and treatment by enrolling a large diverse cohort of at least 1 million persons in the USA. ${ }^{9}$

What is integrative thinking? Roger Martin, a famous Canadian management scientist, once elaborated on the concept of integrative thinking: that is, holding two contradictory views in 


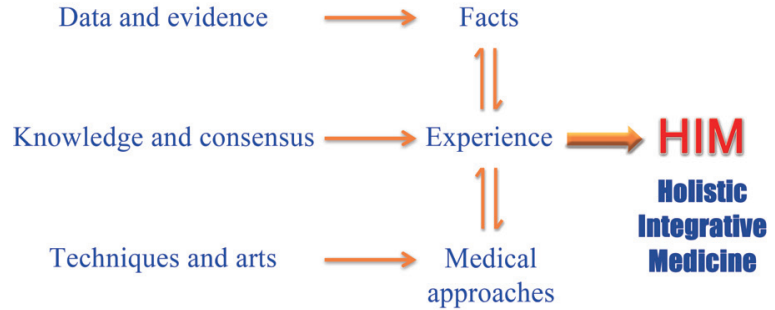

Fig. 1. The connotation and evolvement of holistic integrative medicine (HIM).

the mind at the same time. From this definition, Martin came up with a solution that combines the advantages of both perspectives. This approach aims to deal with opposing views in a constructive way, not at the expense of choosing one over the other, but in an innovative way to eliminate the confrontation between the two views. The new views contain some elements of the opposing points and are superior to both of the original opposing points. ${ }^{10}$ In order to use integrative thinking, it is necessary to grasp several principles: (1) expand the scope of the key factors in decision-making; (2) consider multi-faceted and indirect causality; (3) in the decision-making process, the problem is not divided between several independent individuals to solve one by one, and rather each part is dealt with while maintaining the integrity of the problem. The fourth principle is to struggle to find innovative solutions such that each idea and process is more efficient and accurate than the previous one. For instance, consider an advanced cancer patient is faced with options such as surgery, chemotherapy, radiotherapy, and biological therapy. The order and degree of the treatments and whether they are to be used in combination or sequentially; this requires integrative thinking to make the final decision in the MDT. This is the Holistic Integrative Medicine (HIM) that we have proposed and advocated. ${ }^{4,11}$ Evidence-based medicine (EBM), which has been widely used and established, is not enough alone for MDT. Evidence is the cornerstone of EBM, however, whether an "objective" piece of evidence reflects the truth depends on sample size and when and how the evidence is obtained. Moreover, even the same disease may manifest different symptoms in different patients and may change with time, which is the so-called heterogeneity effect. In the process from considering evidence to decision-making, we should use integrative thinking to see whether the evidence is useful and whether it needs to be used in the patient encountered.

To construct a new medical system more suitable for human health and disease management, HIM regards the human body as a holistic unit. In this sense, this approach organically integrates the most advanced knowledge and theories in each medical field and the most effective practices from various clinical specialties. HIM then develops corresponding revisions and adjustments according to social, environmental, and psychological conditions. ${ }^{4}$ By converting the data and evidence obtained from medical research back into their original facts, transforming knowledge and consensus gained from clinical practice into experience, and consolidating techniques and arts discerned from clinical explorations into medical approaches, HIM takes shape through these repeated practices at the level of facts, experience, and medical approaches (Fig. 1). From MDT to HIM, a multidisciplinary working model should be established to formulate an individualized integrated healthcare plan to achieve an optimal effect using the perspective of HIM.

\section{Acknowledgments}

None.

Funding

This work was supported by the key projects of Chinese Academy of Engineering (No. 2018-ZD-09 and No. 2018-XZ-44).

\section{Conflict of interest}

The authors declare that no conflict of interest exists.

\section{Author contributions}

Review of the literature and drafting of the manuscript (ZY), critical revision of the manuscript (DF), approval for the manuscript (ZY, DF)

\section{References}

[1] Thornton S, Dodwell D. Multidisciplinary team working: the emperor without clothes? Br J Hosp Med (Lond) 2012;73(4):186-187. doi:10.12968/hmed.2012.73.4.186.

[2] Soukup T, Lamb BW, Sevdalis N, Green JS. Streamlining cancer multidisciplinary team meetings: challenges and solutions. Br J Hosp Med (Lond) 2020;81(3):1-6. doi:10.12968/hmed.2020.0024.

[3] Teo C, Broggi M. Surgical outcome of patients considered to have "inoperable" tumors by specialized pediatric neuro-oncological multidisciplinary teams. Childs Nerv Syst 2010;26(9):1219-1225. doi:10.1007/ s00381-010-1199-6.

[4] Fan D. Holistic integrative medicine: toward a new era of medical advancement. Front Med 2017;11(1):152-159. doi:10.1007/s11684017-0499-6.

[5] Quinlan E, Robertson S. Mutual understanding in multi-disciplinary primary health care teams. J Interprof Care 2010;24(5):565-578. doi:10.3109/13561820903520385.

[6] Makary MA, Daniel M. Medical error-the third leading cause of death in the US. BMJ 2016;353:i2139. doi:10.1136/bmj.i2139.

[7] Eigenmann F. Multidisciplinary team meetings encourage overtreatment. BMJ 2015;351:h4630. doi:10.1136/bmj.h4630.

[8] Soukup T, Lamb BW, Arora S, Darzi A, Sevdalis N, Green JS. Successful strategies in implementing a multidisciplinary team working in the care of patients with cancer: an overview and synthesis of the available literature. J Multidiscip Healthc 2018;11:49-61. doi:10.2147/ JMDH.S117945.

[9] All of Us Research Program Investigators, Denny JC, Rutter JL, Goldstein DB, Philippakis A, Smoller JW, et al. The "All of Us" Research Program. N Engl J Med 2019;381(7):668-676. doi:10.1056/NEJMsr1809937.

[10] Martin RL. The Opposable Mind: How Successful Leaders Win Through Integrative Thinking. Boston: Harvard Business School Press; 2007:6.

[11] Fan DM. Holistic Integrative Medicine. Am J Digest Dis 2014;1(1):2236. 